\title{
The role of attitudes and behaviours in explaining socio-economic differences in attainment at age 11
}

\author{
Paul Gregg and Elizabeth Washbrook \\ CMPO, University of Bristol \\ P.Gregg@bristol.ac.uk
}

(Received July 2010 Revised November 2010)

\section{Abstract}

This paper explores the correlates of the socio-economic gradient in children's educational performance through the primary school years. Thus it sits between the companion papers on pre-school cognitive outcomes and attainment in the secondary school years in this Special Issue. The poorest $20 \%$ of children score, on average 14 percentile points lower than the middle 20\% in Key Stage 2 tests at age 11, and 31 percentile points lower than the richest 20\%. Overall around one third of the attainment gaps by socio-economic background at age 11 are found to emerge after age 7. The evolution of attainment gaps over this period is found to be related a range of attitudes to education and behavioural patterns of the study children. Low maternal aspirations for the child's final educational attainment are strongly linked to the widening socio-economic gap during these years, over and above their influence on the child's own measured attitudes and behaviours.

JEL codes: 121

Key words: education, inter-generational transmission, socio-economic gap

\section{Introduction}

Children growing up in less affluent families emerge from our schools with substantially lower levels of educational attainment. These educational deficits emerge early in children's lives, even before entry into school, and widen throughout childhood (see Feinstein 2003 and 2004). This paper focuses on the differences between socio-economic groups in academic performance at the time of entry into secondary education at age 11 . We use data from the Avon Longitudinal Study of Parents and Children (ALSPAC), a cohort of around 14,000 children born in Avon in the early 1990s. ${ }^{i}$ In ALSPAC, the $20 \%$ of children with the lowest socio-economic position score, on average, 14 percentile points lower than the middle 20\% in Key Stage 2 tests at age 11, and 31 percentile points lower than the richest $20 \%$. Children from low socio-economic backgrounds perform less well on tests at age 7. Moreover, poor children who perform well at 7 are more likely than rich children to have slipped back by age 11, and poor children who perform badly at 7 are less likely to catch up over the period. Around one third of the attainment gaps by socio-economic background at age 11 emerge after age 7.

We aim to examine some of the routes through which family socio-economic position (SEP) affects educational attainment. Our focus is on a range of early parenting behaviours and on parent and child attitudes, behaviours and 
beliefs in the primary years, that have seldom been studied in work on the causes and consequences of poverty. We aim to explore which attitudes and beliefs are important in influencing attainment at age 11 and patterns of educational development between the ages of 7 and 11, and the relative strength of these influences. Parental and child attitudes and beliefs are likely to be correlated with a range of other family background factors and hence it is difficult to disentangle the independent influence of the mechanisms of interest. We seek to minimise this problem in three ways. First, we adopt a distal/proximal modelling approach in which a range of family demographic measures 'compete' with our posited transmission mechanisms, to explain educational patterning by socio-economic background. Measures such as parental education, age and number of siblings are included to 'mop up' any correlated but unobserved influences on educational attainment. School characteristics are also included as proxies in this way. Second, we explore the extent to which attitudes and behaviours are associated with educational development over the four years prior to the start of secondary schooling, by including measures of attainment at age 7 . The inclusion of prior attainment, focuses the estimates on development during the specified window, and gives an insight into how much of the influence of attitudes and behaviours are already crystallized in attainment at the start of junior school. A final approach is to include earlier measures of parenting and the home learning environment which influence attainment at age 5 (see Dearden et al in this Issue), and again help to isolate the role of transmission mechanisms during the primary school years. If early (pre-school) environments are the major determinants of educational trajectories, then omitting them from our analysis may falsely overstate the role of the attitudes and behaviours in middle childhood that are our focus. Despite these strategies, the danger that our estimates pick up the correlation of the specified transmission mechanisms with unobserved factors remains. However, it should not be assumed that our estimates necessarily overstate the potential contribution of attitudes and behaviours to the socioeconomic gap. Any mis-measurement of the complex psychological constructs in question will have the reverse effect, and tend to bias the estimated associations downward.

\section{Modelling approach}

The aim of our analysis is to better understand the observed relationship between a child's socio-economic background and his or her educational performance at 11 . In particular, the aim is to assess the importance of attitudes and aspirations, both of the parents and the child, on attainment. We explore the role of a diverse range of factors that potentially mediate this relationship, in the sense that they are correlated with family background and directly influence children's educational development. The ALSPAC data is extremely rich, so we organise our data according to distinct concepts that have been identified in the literature. The broad distalproximal modelling framework is laid out in the overview paper in this Special Issue by Goodman et al (2010), section 4 and in particular in Figure 3. Socio-economic position (SEP) is the principal family background indicator of interest. However, conceptually it is only one of a set of 'distal' factors, or aspects of family background, that together characterise the social and cultural resources available to the child. SEP, along with parental education, family structure and size, school quality and other distal variables, are assumed to shape the 'proximal' attitudes, behaviours and beliefs that are the focus of this study. We hypothesise that the values and behaviours of parents (both in the pre-school period and during the primary school years) and of the children themselves, are relatively more immediate or direct influences on educational achievement, and help to explain why we 
observe differences in average Key Stage scores between different distal groups.

Our choice of potential mediating processes is informed by data availability and by a diverse social science literature on the determinants of educational success. Many of the concepts used in this study are laid out in the overview paper (Goodman et al 2010). In this study, we draw in particular on concepts informed by the expectancy-value theory of achievement motivation, proposed by Eccles, Wigfield and colleagues (e.g. Wigfield and Eccles 2000). Underlying this theory, is the idea that individuals' achievement-related choices, persistence and performance in an activity can be explained by their beliefs about how well they will do the activity, and the extent to which they value the activity. However, we do not restrict our attention solely to motivational constructs, but also consider the role of factors such as children's behavioural problems, or self-regulation, which have been linked to educational performance in a largely unrelated literature (see for example, McLeod and Kaiser 2004; Currie and Stabile 2006). A child's degree of self-regulation influences the ability to see intentions through, and thus interacts with achievement motivation in influencing the expected outcome. The relationship between the parents' and child's motivations and expectations is also likely to be complex. The parent will be aware, though potentially imperfectly, of the child's capabilities, and will adjust expectations accordingly. However, the parents' own experiences, and those of others they are aware of, may also shape their own expectations of the child and their efforts to influence the child's motivation and decisionmaking.

In our analysis, we present results on the relative importance of each transmission mechanism, with and without conditioning on Key Stage 1. The first set of results (the levels model) reveals how far each factor is associated with Key Stage 2 outcomes, without distinguishing how far its influence is manifested pre- or post-age 7 . The second set of results (the value-added model) holds child attainment at 7 constant, and hence reveals how far each factor is associated with improvement or deterioration of the child's performance during the primary school years. In effect, the value-added model measures the influence of covariates on relative progress between the ages of 7 and 11. It addresses the question of whether and where gaps would open up during the period even if all children started with the same level of academic ability. The formal representation of the estimation structure and the results decompositions are laid out in the overview paper, to reduce repetition within each paper in the series.

\section{Data and measures}

ALSPAC is a cohort study that recruited around 14,000 pregnant women who were resident in the Avon area of England whose expected date of delivery fell between $1^{\text {st }}$ April 1991 and $31^{\text {st }}$ December 1992 . Study families were surveyed via high frequency postal questionnaires from the time of pregnancy onwards, and via a number of hands-on clinics in which ALSPAC staff administered a range of detailed physical, psychometric and psychological tests to the children. ALSPAC has been linked to the National Pupil Database (NPD), which contains school identifiers and results on national Key Stage school tests for all children in the public school system, and information of local deprivation at the small area level (the government-produced Indices of Multiple Deprivation, IMD). For details of the comparability of ALSPAC with national data and attrition and sample selection issues for data used in the study please refer to Appendix 1 in the Supplementary Files. ii

The key outcome variable in our analysis is derived from the results of Key Stage 2 assessments in English, maths and science, taken by all pupils in state schools in Year 6 (age 11). We construct an average measure of performance on the three tests, and express this total as a percentile score. We explore two specifications of the Key Stage 2 outcome in all 
the main results in the paper: one without, and one with, a control for Key Stage 1 performance at age 7. Again, the Key Stage 1 measure is constructed as an average score, here over reading, writing and maths tests.

Our measure of socio-economic position is derived from data on a number of indicators: household income at age 2 to 3 , age 7 and age 11 (3 variables), mother's and father's social class ( 2 variables), housing tenure at ages 2 to 5 ( 2 variables) and average reported experience of financial difficulties at ages 0 to 7 (1 variable). We extract the first principal component using the method described in Goodman et al (2010), a component that accounts for $48 \%$ of the variation in the component variables, and individuals are then placed into quintiles (fifths) of the population ranked by this measure. The aim is construct a long-term measure of the material resources of the household, one that incorporates the fact that deprivation is multi-dimensional and that socio-economic risk factors are likely to be cumulative.

Many of our measures of potential transmission mechanisms are taken from a mother-reported postal questionnaire when the child was 9 years old, and from a hands-on clinic when the child was 8 . The timing on these measures is advantageous, because they occur between the Key Stage 1 and Key Stage 2 tests. This enables us to explore how different family environments affect the trajectory of a child's development, given their prior attainment. However, the data requirements are such that families must have remained in the study from birth to 9 years. This is quite a stringent requirement and there is substantial attrition, leaving us with a working sample of only 7972 , about half of original cohort remaining in English state schools. We have used a number of techniques to ensure that our definitions of SEP groupings, and the scaling of the Key Stage outcome variables, are as representative as possible of the national population, rather than only those who remain in the sample (see the appendix A2). Missing values on the explanatory variables are dealt with by mean replacement and the inclusion of a missing dummy.

The ALSPAC data contain information on a wide range of factors that may help to explain the poorer educational performance of sociallydisadvantaged children. We distinguish two types of explanatory factors. First are 'distal' factors that describe the resources available to parents and children in a broad sense, and capture the structural features of the environments in which children are raised.

Parental education consists of variables that capture the mother's and father's highest educational qualification, measured just prior to the child's birth (Certificate of Secondary Education/no qualifications; Vocational/O-level; Alevel; Degree).

Demographic characteristics consist of variables measuring child's gender; ethnicity (dummy for non-white); month of birth (scaled to September $=0$ ); family structure at age 7 (resident biological father, step-father, or single parent); mother's age at birth (dummies for <20; 20-24; 25-29; 30-34; 35 or more); number of older siblings (none, 1, 2, 3 or more); number of younger siblings at age 9 (none, 1, 2 or more); mother's and father's employment status at age 4; an indicator for English as a second language; and mother's and father's self-rated health (on a scale of 1 to 4 ) at age 4 .

School characteristics are the mean Key Stage 1 score of all pupils at the child's school; the mean value-added between Key Stages 1 and 2 of all pupils at the child's school (both standardized to unit variance); and the proportion of pupils in the school eligible for free school meals (FSM).

The next groups of variables are the attitudes, behaviours and beliefs of parents and children or the 'proximal' factors, that we expect to be shaped by the distal features of the family's circumstances, and that in turn directly affect the learning process.

Pre-school environments consists of variables relating to health, cognitive stimulation and childcare experiences, all 
measured prior to age 5. These bear a reasonably close relationship with those used in the companion early years paper in this issue by Deaden et al (2010). Our analysis allows us to explore whether these early influences have an association with faster progress between 7 and 11, perhaps because they capture differences between parents that persist. Included variables are: birth weight (in $\mathrm{kg}$ ); an indicator of gestation less than 37 weeks; indicators of breastfeeding duration (never, less than 3 months, 3 to 6 months, or more than 6 months); an indicator for whether the mother smoked in pregnancy; an indicator for post-natal depression (average Edinburgh Postnatal Depression Scale over six dates, from pregnancy to 33 months ); quintile groups of a Home Learning Environment (HLE) index (average of 9 standardized items measured at age 3: frequency that child is taken to the library, frequency that mother reads and sings to the child; indicators for whether the mother tries to teach the child colours, the alphabet, numbers, nursery rhymes, songs, and shapes and sizes); indicators for whether the child is read to every day and has a regular sleeping routine at 3; indicators for whether the child regularly attended a day nursery or crèche before age 3 , and attendance at a nursery at age 3 or 4 .

Other parental attitudes and behaviours consider potential ways in which parents may influence the educational development of children during the primary school years. These include a measure of the mother's sense of control in life (the 12-item Adult NowickiStrickland Locus of Control scale, standardized to unit variance); the mother's rating of whether school was a valuable experience for her (5-point scale, standardized to unit variance); the mother's aspirations for her

lifting, drinking alcohol or carrying a weapon, each standardized and averaged).

Other contexts besides the home may also impact on ability and motivation. Here we examine experience of bullying (frequency of occurrence of nine events in the last 6 months, child's education (get good GCSEs then leave, take at least one A-level; go to university, other); the amount and quality of mother-child educational interactions likely to contain some component of cognitive stimulation (eight 5point items such as, helps with homework and draws or paints with child, standardized and averaged); and the amount and quality of other mother-child non-educational interactions that may nevertheless be important in fostering family bonds and socio-emotional well-being in children (nine 5-point items such as takes child to the park and does active play with child, standardized and averaged).

Child's attitudes and behaviours are captured by three broad groupings of variables. Beliefs and values include the child's perceptions of their own ability (the scholastic competence sub-scale of Harter's SelfPerception Profile for Children, standardized to unit variance); their feelings about, or intrinsic valuation, of school (the average of 15 standardized items such as how much the child enjoys school, seems bored by school and enjoys different lessons); their sense of what is important in life, or extrinsic values (indicators of whether the child believes school results, hobbies and interests, and material possessions to be important in life); and their sense of how personal effort in general will impact on their lives (the 12-item Nowicki-Strickland InternalExternal Scale, standardized to unit variance).

Behavioural difficulties, which may interfere with academic progress, are measured in terms of symptoms of hyperactivity, conduct problems and emotional problems (three subscales of the parent-report Strengths and Difficulties Questionnaire, each standardized to unit variance); and engagement in anti-social activities (11 binary indicators for whether the child has ever engaged in behaviours like shopsuch as having belongings taken, being threatened and being called names, standardized and averaged); experience of other peer problems (the Strengths and Difficulties Questionnaire peer problems subscale); participation in out-of-school activities, 
such as sports, singing or drama groups (eight items capturing the frequency of participation, standardized and averaged); other pro-social behaviours (the Strengths and Difficulties prosocial sub-scale); and teacher-child relations (six items such as whether the child is frightened of the teacher and whether he/she believes the teacher thinks his/her work is good, standardized and averaged).

\section{Results}

\subsection{Socio-economic differences in child and family characteristics}

The left hand panel of Figure 1 shows the average test scores of children in our working sample at age 11 (KS2), by quintile of SEP. The scores reflect the child's percentile in the Avon distribution and range from 1 , for the lowest performing children, to 100 for the highest performing. If there were no systematic differences in attainment by SEP, each group would have an average score of 50.5. Deviations from this number show how far children from different socio-economic backgrounds tend to over- or under-perform relative to the average. It is clear that there are substantial differences in educational performance that run throughout the socioeconomic scale. The mean score of the most advantaged children is 31 percentile points higher than that of the most disadvantaged.

Figure 1. Test scores at 11 by parental SEP quintile
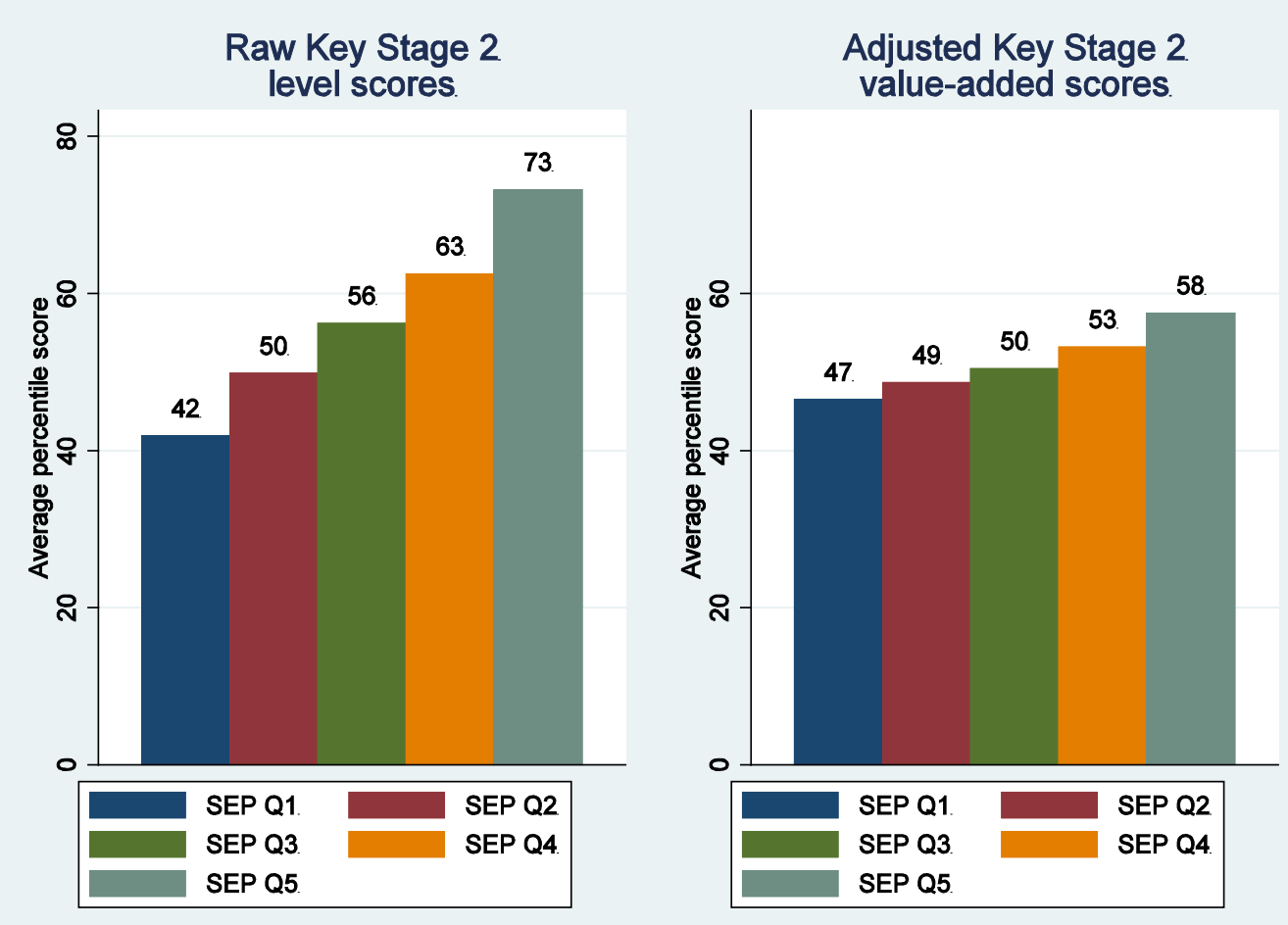

Note: the right hand panel presents an 'adjusted' gap, showing the average percentile score by SEP quintile, assuming all children scored the same at age 7. Such estimates are derived by predicting each individual's Key Stage 2 percentile in the situation where all pupils scored equally (i.e. at percentile 50.5) at Key Stage 1, based on a 'value-added' regression of the following form: $K S_{i 11}=\alpha+\lambda S E P_{i}+b K S_{i 7}+\varepsilon_{i 11}$.

Although there are some differences around the middle of the SEP distribution, it is noticeable that the largest gaps occur at the tails, with the poorest children falling far behind, and the richest children pulling strongly ahead. The right hand panel of Figure 1 explores how these patterns are affected by controlling for prior attainment at Key Stage 1 (KS1; age 7). The gradients here are noticeably smaller than in the left panel, and show that a 
large fraction of the educational inequality observed at age 11 in fact reflects differences that are already apparent by age 7 . However substantial differences remain, and suggest that the poorest children fall a further 11 percentile points (almost one-third of the raw 31 point gap) behind the richest children between 7 and 11, even if they started the period with the same levels of attainment.

Table 1 explores the trajectories of children from different socio-economic groups in more detail. The first two rows focus on lowachieving children at age 7. The lower SEP children in our sample are much more likely to fall into this group $-54 \%$ of the bottom quintile scored at the $40^{\text {th }}$ percentile or below, compared with only $16 \%$ of the top quintile. They are also much less likely to escape from the low-achieving group by age 11 . Less than a quarter of the poorest children who scored in the bottom $40 \%$ at age 7 , are able to escape by age 11 , whereas more than half of those in the top SEP quintile are no longer there four years later. The next two rows show a parallel pattern for the high-achieving children at 7 . Low SEP children are much less likely to be scoring above the $60^{\text {th }}$ percentile at this age ( $25 \%$ of the poorest compared with $64 \%$ of the richest), and those who do are more likely to have dropped out of this high-achieving group by age 11 (28\% of the poorest fall back compared with only $8 \%$ of the richest highachievers). It is the combination of these two factors - that low SEP children start behind at age 7 and that high achieving children from poorer families do not progress as well as higher SEP children and are often overtaken by less able more affluent children during the primary school years - that results in the patterns shown in the final two rows of the table. At 11, children in the bottom SEP quintile are nearly five times as likely to be lowachievers, and two-and-a-half times less likely to be high-achievers, as children in the top SEP quintile.

Table 1. Educational performance at 7 and 11, by parental SEP

\begin{tabular}{|c|c|c|c|c|c|}
\hline & SEP & SEP & SEP & SEP & SEP \\
\hline Proportion of children: & Q1 & Q2 & Q3 & Q4 & Q5 \\
\hline Bottom $40 \%$ at KS1 & 0.54 & 0.41 & 0.33 & 0.27 & 0.16 \\
\hline Escape from bottom $40 \%$ by $K S 2^{a}$ & 0.23 & 0.29 & 0.32 & 0.37 & 0.54 \\
\hline Top $40 \%$ at KS1 & 0.25 & 0.34 & 0.42 & 0.50 & 0.64 \\
\hline Dropout of top $40 \%$ by KS2 ${ }^{a}$ & 0.28 & 0.26 & 0.18 & 0.14 & 0.08 \\
\hline Bottom $40 \%$ at $\mathrm{KS} 2$ & 0.51 & 0.38 & 0.31 & 0.24 & 0.11 \\
\hline Top $40 \%$ at $\mathrm{KS} 2$ & 0.28 & 0.37 & 0.47 & 0.57 & 0.73 \\
\hline
\end{tabular}

Top and bottom 40\% refer to the proportions in the population of all children in the Avon area. Proportions in the working sample do not equal exactly 0.40 because the working sample is positively selected, and because Key Stage 1 scores are only semi-continuous. 7972 observations. 
The characteristics of low income families differ from those of their more affluent counterparts along many dimensions. Table 2 documents the socio-economic gaps in each of our potential transmission mechanisms. The first panel focuses a number of dimensions of the pre-school environment that plausibly affect children's health and development. Breast feeding, maternal smoking and post-natal depression are all very strongly graded by SEP, and there are also some differences in average birth weight and the likelihood of being born pre-term. Less advantaged parents tend to engage in fewer reading and teaching behaviours with their three-year-olds, although this is not universally true: $13 \%$ of the poorest families are in the highest home learning environment quintile, while $14 \%$ of the most affluent families are in the bottom quintile. Finally, we see that exposure to centre-based child care before the age of 3 was relatively rare in this cohort, but much more common among the better off, while attendance at nursery at ages 3 to 4 was most common among the best and worst off, with middle SEP children showing the lowest participation rates. We might imagine that the quality of child care settings would differ with family income, for example because the most advantaged can afford expensive private nurseries ${ }^{\text {iii. }}$. Research suggests that quality is the key factor that determines the influence of childcare on children's development (NICHD ECCRN and Duncan 2003), but unfortunately we are not able to measure it here, and can only explore differences in exposure to particular types.

Table 2. Means of mechanism variables, by quintile of socio-economic position

$\begin{array}{cccccc}\text { Poorest } & 2^{\text {nd }} \text { SEP } & \text { Middle } & 4^{\text {th }} \text { SEP } & \text { Richest } & \text { Q5-Q1 } \\ \text { SEP } & \text { quintile } & \text { SEP } & \text { quintile } & \text { SEP } & \\ \text { quintile } & \text { (Q2) } & \begin{array}{c}\text { quintile } \\ \text { (Q1) }\end{array} & \text { (Q4) } & \text { quintile } & \\ \text { (Q1) } & & \text { (Q3) } & & \text { (Q5) }\end{array}$

Parental attitudes and behaviours

\section{A. Pre-school environments}

Birth weight $(\mathrm{kg})$

3.34

3.40

3.44

3.44

3.46

$0.11 \mathrm{~kg}^{* *}$

Gestation < 37 weeks

$6.4 \%$

$6.4 \%$

$5.4 \%$

$5.6 \%$

$4.4 \%$

$-1.9 \mathrm{ppt}$

Breast fed: Never

$46.3 \%$

$34.1 \%$

$27.3 \%$

$21.1 \%$

$10.6 \%$

$24.9 \%$

Breast fed: 3-6 mths

$11.3 \%$

$25.9 \%$

$26.4 \%$

$25.9 \%$

$18.0 \%$

$17.5 \%$

$14.5 \%$

$18.0 \%$

$17.6 \%$

$18.7 \%$

$-35.7 \mathrm{ppt}^{* *}$

Breast fed: 6 mths +

$28.3 \%$

$35.4 \%$

$52.7 \%$

$-6.9 \mathrm{ppt}^{* *}$

$56.5 \%$

$38.3 \%$

$23.2 \%$

$18.3 \%$

$10.7 \%$

Mother had post-natal depression

$27.3 \%$

$18.6 \%$

$12.0 \%$

$10.1 \%$

$8.1 \%$

$30.6 \%$

$24.0 \%$

$19.6 \%$

$15.7 \%$

$13.8 \%$

HLE at 3: Second quintile

$23.3 \%$

$22.9 \%$

$22.0 \%$

$21.0 \%$

$17.3 \%$

$7.4 \mathrm{ppt}^{* *}$

HLE at 3: Middle quintile

$18.6 \%$

$20.6 \%$

$19.6 \%$

$21.1 \%$

$19.6 \%$

$35.2 \mathrm{ppt}^{* *}$

$14.0 \%$

$15.1 \%$

$19.0 \%$

$21.6 \%$

$23.4 \%$

$-45.9 \mathrm{ppt}^{* *}$

HLE at 3: Highest quintile

$13.4 \%$

$17.5 \%$

$19.8 \%$

$20.6 \%$

$25.9 \%$

$-19.2 \mathrm{ppt}^{* *}$

$52.3 \%$

$59.7 \%$

$63.7 \%$

$66.8 \%$

$71.4 \%$

Child has regular sleep routine at 3

$85.2 \%$

$89.7 \%$

$91.9 \%$

$94.7 \%$

$96.5 \%$

$-16.9 \mathrm{ppt}^{* *}$

$7.2 \%$

$9.3 \%$

$9.8 \%$

$15.7 \%$

$24.2 \%$

$-6.0 \mathrm{ppt}^{* *}$

$40.4 \%$

$38.1 \%$

$35.7 \%$

$44.0 \%$

$53.6 \%$

$1.0 \mathrm{ppt}$

9.4 ppt** $^{* *}$

$12.5 \mathrm{ppt}^{* *}$

$19.1 p p t * *^{* *}$

$11.3 \mathrm{ppt}^{* *}$

$16.9 \mathrm{ppt}^{* *}$

$13.2 \mathrm{ppt}^{* *}$ 
(Table 2 cont'd)

\section{B. Other attitudes and behaviours}

Mother found school valuable

(scale)

Mother hopes child will get good

GCSEs

Mother hopes child will get at

least 1 A-level

Mother hopes child will go to

university

Mother hopes other for child

Mother-child interactions:

Education (scale)

Mother-child interactions: Non-

educational (scale)

$\begin{array}{cccccc}-0.64 & 0.28 & 0.01 & 0.27 & 0.61 & 1.26 \mathrm{sd}^{* *} \\ -0.39 & 0.21 & -0.01 & 0.1 & 0.35 & 0.74 \mathrm{sd}^{* *} \\ & & & & & \\ 21.9 \% & 16.2 \% & 11.3 \% & 6.4 \% & 1.4 \% & -20.5 \mathrm{ppt}^{* *} \\ & & & & & \\ 19.0 \% & 20.4 \% & 19.0 \% & 14.2 \% & 6.6 \% & -12.4 \mathrm{ppt}^{* *} \\ & & & & & \\ 36.5 \% & 41.4 \% & 50.2 \% & 62.4 \% & 80.5 \% & 44.0 \mathrm{ppt}^{* *} \\ 22.7 \% & 22.1 \% & 19.5 \% & 17.0 \% & 11.5 \% & -11.2 \mathrm{ppt}^{* *} \\ & & & & & \\ 0.03 & 0.01 & 0.02 & 0.00 & -0.03 & -0.06 \mathrm{sd}^{* *} \\ -0.03 & 0.01 & 0.02 & 0.01 & -0.01 & 0.02 \mathrm{sd}\end{array}$

Child's attitudes and behaviours

\begin{tabular}{|c|c|c|c|c|c|c|}
\hline Ability beliefs (scale) & -0.12 & 0.11 & -0.06 & 0.07 & 0.11 & $0.24 \mathrm{sd}^{* *}$ \\
\hline Locus of control (scale) & -0.31 & 0.16 & -0.1 & 0.07 & 0.28 & $0.59 \mathrm{sd}^{* *}$ \\
\hline Enjoyment of school (intrinsic & & & & & & \\
\hline values, scale) & -0.03 & 0.03 & 0 & 0.04 & 0.02 & $0.06 \mathrm{sd}$ \\
\hline $\begin{array}{l}\text { School results important in life } \\
\text { (extrinsic values) }\end{array}$ & $50.6 \%$ & $55.8 \%$ & $61.3 \%$ & $62.9 \%$ & $66.6 \%$ & $16.0 \mathrm{ppt}^{* *}$ \\
\hline $\begin{array}{l}\text { Hobbies important in life } \\
\text { (extrinsic values) }\end{array}$ & $66.6 \%$ & $70.8 \%$ & $75.2 \%$ & $77.3 \%$ & $83.5 \%$ & $16.9 \mathrm{ppt}^{* *}$ \\
\hline $\begin{array}{l}\text { Possessions important in life } \\
\text { (extrinsic values) }\end{array}$ & $75.0 \%$ & $76.6 \%$ & $76.7 \%$ & $76.5 \%$ & $78.5 \%$ & $3.6 \mathrm{ppt}^{*}$ \\
\hline Anti-social behaviours (scale) & 0.1 & 0.03 & -0.01 & -0.02 & -0.03 & $-0.13 s d^{* *}$ \\
\hline Hyperactivity (scale) & 0.27 & 0.15 & -0.02 & -0.08 & -0.16 & $-0.42 \mathrm{sd}^{* *}$ \\
\hline Emotional symptoms (scale) & 0.17 & 0.06 & -0.02 & -0.03 & -0.1 & $-0.27 \mathrm{sd}^{* *}$ \\
\hline Conduct problems (scale) & 0.33 & 0.11 & -0.04 & -0.08 & -0.14 & $-0.47 s d^{* *}$ \\
\hline Experience of bullying (scale) & 0.08 & 0 & 0 & 0 & -0.06 & $-0.14 \mathrm{sd}^{* *}$ \\
\hline Pro-social behaviours (scale) & -0.04 & 0 & 0.04 & 0.04 & -0.05 & $-0.01 \mathrm{sd}$ \\
\hline Peer problems (scale) & 0.32 & 0.08 & -0.04 & -0.07 & -0.12 & $-0.44 s d^{* *}$ \\
\hline $\begin{array}{l}\text { Leisure/out-of-school activitie } \\
\text { (scale) }\end{array}$ & -0.23 & 0.16 & -0.07 & 0.04 & 0.22 & $0.45 \mathrm{sd}^{* *}$ \\
\hline child & .04 & 0.01 & 0.02 & 0.02 & 0.01 & 05 sc \\
\hline
\end{tabular}

Full sample contains 7972 observations. Means defined over non-missing responses only. Q1 denotes the lowest SEP quintile, Q2 the second lowest SEP quintile, and so on; HLE denotes the home learning environment index; ppt denotes percentile points; scale denotes the average of a number of standardized item scores; std score denotes a variable standardized to mean 0 , standard deviation 1 on the maximum available sample; sd denotes standard deviations. ** and * indicate significance at the 1 and $5 \%$ levels respectively.

The second panel of Table 2 highlights differences in other parental attitudes and behaviours that are not specific to the pre-school period. Low SEP mothers tend to have a much more external locus of control (a sense that luck or fate, rather than their own actions, are what matters in life), and tend to view their own schooling experiences as having been less valuable than more advantaged mothers. Most strikingly, there are very large differences in their educational aspirations for their children when they are age $9.81 \%$ of mothers in the richest 
quintile hope their child will go to university, compared with only $37 \%$ of mothers in the lowest quintile. Interestingly, these differences do not appear to be mirrored in differences in the frequency and variety of mother-child interactions at 9. We find only very small differences in mothers' reports of how often they make things or read with the child, help with homework, etc (educational interactions), and in how often they take the child to the park or shopping, prepare food with the child, etc (noneducational interactions). Hence the marked socio-economic differences we see in pre-school parenting behaviours seem to have narrowed by the mid-primary school years.

The final panel in Table 2 shows how children's own attitudes, behaviours and beliefs differ with socio-economic position. There are notable differences in the degree to which lowincome children express negative views at the ages of 8 to 9. They tend to regard themselves as scholastically less able, they are less likely to view school results as important in life (as reported by the mother) and tend to have a more external locus of control. This sense that luck or chance determines outcomes, rather than one's own efforts, is also found among low income mothers, but the gradient is much less marked in the children than in their mothers. In contrast, we find little differences in the extent to which children enjoy school or value it for its own sake, and in the likelihood of believing material possessions to be important in life. Low income children are much more likely to exhibit behavioural problems in terms of hyperactivity, conduct problems and peer relations, including being a victim of bullying, whereas pro-social (cooperative) behaviours and teacher-child relations differ less with family background. Finally there is evidence of marked differences in participation in out-of-school leisure activities such as sports, clubs and classes.

\subsection{Explaining the socio-economic gaps in educational outcomes at 11}

So far we have shown that parental and child aspirations, attitudes and behaviours differ across socio-economic position of the family. These analyses, however, do not show how important attitudes and behaviours are to attainment overall, nor do they identify which aspects of our range of measures of parent and child behaviours and beliefs contribute most to the transmission of socio-economic position. In this section, we explore which factors do the heavy lifting in explaining SEP attainment gradients in children, and the relative importance of specific attitudes, behaviours and beliefs. The explanatory power of a given variable depends on two associations: the extent to which it varies by socio-economic group, and the extent to which it independently predicts educational outcomes (see the overview paper by Goodman et al. 2010). Factors that vary little with socioeconomic position - such as parent-child interactions at age 9 - cannot play an important role in generating the social gradient in outcomes. This does not imply, however, that such factors are not consequential for educational achievement in general. Equally, a factor may be strongly socially graded, but if it has little association with outcomes, its role in explaining the gradient will be small. In the search for the key attitudes and behaviours that drive the observed SEP gaps, we need to identify factors that are both concentrated in disadvantaged families, and that strongly interfere with the development of children's learning. Table 2 gave the levels of patterning of variables by SEP, so next we focus on the relationship between behaviours and educational attainment in a fully conditioned model. This provides estimates of the direct effects of each of the individual variables, over and above other aspects of family background.

We explore this in two models (levels and value-added), reporting the marginal association of each individual measure of attitudes and behaviours with Key Stage 2, given all other aspects of family background. This analysis allows us to identify factors that are not, or only weakly, socially graded but that may nevertheless be consequential for 
educational achievement. Table 3 shows selected coefficients with the focus on attitudes and behaviours rather than family background characteristics, from two fullycontrolled regression models of Key Stage 2 (i.e. conditioning on all the variables listed in section 3), with the sole difference between the two being the inclusion of Key Stage 1 control in column 2 . Looking first at the influence of pre-school environments, we find significant associations of the expected sign between Key Stage 2 and birth weight and gestation, breastfeeding, and the home learning environment at 3 , which is notable given the rich set of other controls included in the model. Birth weight and the pre-school learning environment become insignificant when Key Stage 1 is added to the model, implying that their influence on academic ability is fully apparent by age 7. Two 'perverse' results are the positive association of pre-natal smoking, and the negative association of reading to the child daily at age 3 with Key Stage 2. Both of these factors appear uncorrelated with Key Stage 1, as the associations remain strong when prior ability is controlled. We cannot say conclusively why these patterns arise, but it perhaps an illustration of the dangers of an 'overcontrolled model' discussed in the overview chapter, where strong co-linearities between the covariates result in the identification of individual marginal effects from rare and unrepresentative observations. Here, early reading is strongly related to the home learning environment and suggests that this is the key factor. The home learning environment is highly significant and materially important to attainment at age 11 , but this is entirely captured by attainment at age 7 and does not contribute to the value-added models. This is strong evidence for this being an age-specific factor, rather than a marker for time-invariant, unobserved, positive parental characteristics.

Looking next at maternal attitudes and behaviours during primary school, we see that maternal locus of control is a significant predictor of age 11 outcomes, because it is associated both with higher ability at 7 and faster progress between 7 and 11 . The magnitude of the association between maternal aspirations and child outcomes is, however, far more dramatic. Holding all else constant, children of mothers who hope they will go to university, score 13.7 percentile points higher at Key Stage 2 than children of mothers who want them to get good GCSEs then leave. This can be contrasted the gap of 4.5 between the lowest and the highest SEP quintiles estimated in the same regression. More than half of the effect of maternal aspirations is absorbed when we control for Key Stage 1, but maternal aspirations remain one of the biggest single predictors of progress between 7 and 11. It is not the case, then, that maternal aspirations simply reflect the child's revealed level of ability from school tests two years previously, although they are related. These findings are particularly strong given that they are direct effects, that is, net of any indirect effects on outcomes via children's own attitudes and behaviours.

Virtually all of our variables capturing children's attitudes, behaviours and beliefs have independent, statistically significant, if modest associations with Key Stage 2 outcomes. Children's beliefs in their own scholastic ability and their locus of control, are associated partly with faster progress between 7 and 11, but also reflect higher ability as measured at age 7 . This may be because educational success promotes positive attitudes, but it may also be that positive attitudes begin earlier in life, and that this in part determines attainment at 7. A positive intrinsic valuation or enjoyment of school is also associated with higher scores at 11 , but this effect is entirely explained by the higher prior achievement of children with positive valuations of school. Extrinsic values, however, or beliefs that something is important in life, continue to influence learning after the age of 7. Children who believe that school results or hobbies and interests are important in life, 
score better at both ages, while those that believe material possessions to be important tend to fall behind. We find further evidence that hobbies and interests are associated with educational achievement, as the frequency of participation in leisure activities (such as sports, singing/drama lessons and groups such as Scouts) is a significant predictor of the outcome, although again only because it is positively correlated with prior achievement.

Children's social and behavioural skills are indeed key factors explaining educational success. Hyperactivity or attention problems are particularly associated with adverse outcomes, as are conduct problems and antisocial behaviours (fighting, stealing, substance abuse, etc). In all these cases controlling for prior attainment does not eliminate the effect, which would appear to be evidence against a hypothesis that they are simply correlated with low cognitive ability. Instead our results provide support for the idea that behavioural difficulties interfere with the learning process over the course of primary school and lead to under-performance at age 11. Interestingly, we find no association between emotional symptoms (which relate more to depression, anxiety and internalising behaviours) and performance at either 7 or 11 . And pro-social and peer relations have the opposite effects than expected. Children who exhibit strongly pro-social behaviours (e.g. volunteering to help others, sharing readily with other children) and also those who experience fewer problems with peers (e.g. tending to be liked by other children and spending time with them) score worse at Key Stage 1 and progress more slowly thereafter than more self-contained solitary children. These results are interesting because they highlight that different types of social and behavioural problems differ strongly in their association with educational performance.

Overall it is worth noting that the fit of these models is high, especially when age 7 attainment is included. In the model for age 11 attainment, just under half the total variance is explained, and including lagged age 7 attainment raises this to $70 \%$. This suggests that the models being described do capture, to a large degree, the variance in child attainment, even if it is inevitable that some factors will be poorly measured.

Table 3. Estimated effects of attitudes, behaviours and beliefs on Key Stage 2 scores

Regression coefficient

\section{Variable}

Pre-school environments

Birth weight $(\mathrm{kg})$

Gestation < 37 weeks

Breast fed: Never

Breast fed: $<3$ mths

Breast fed: 3-6 mths

Breast fed: 6 mths +

Mother smoked in pregnancy

Mother had post-natal depression

HLE at 3: Lowest quintile

HLE at 3: Second quintile

HLE at 3: Middle quintile

HLE at 3: Fourth quintile

HLE at 3: Highest quintile

Child read to dailv at 3

Child has regular sleeping routine at 3
(1)

(2)

$\begin{array}{cc}2.0 * * * & 0.5 \\ 2.0 * & 1.5^{*}\end{array}$

\begin{tabular}{cc}
\multicolumn{3}{c}{ Omitted } \\
0.1 & 0.1 \\
0.8 & 0.9 \\
$1.9^{* * *}$ & $1.9 * * *$ \\
& \\
$1.3^{* *}$ & $0.9 *$ \\
0.5 & 0.5 \\
& \\
& Omitted \\
$1.3^{*}$ & \\
$4.1^{* * *}$ & -0.2 \\
$4.6^{* * *}$ & 0.7 \\
$5.2^{* * *}$ & 0.2 \\
& 0.4 \\
$-2.2^{* * *}$ & \\
0.8 & $-1.4 * * *$ \\
&
\end{tabular}

0.8

0 


\section{Variable}

Centre-based child care pre-age 3

Nursery age 3 to 4

Other parental attitudes and behaviours

Mother's locus of control (scale)

Mother found school valuable (scale)

Mother hopes child will get good GCSEs

Mother hopes child will get at least $1 \mathrm{~A}$-level

Mother hopes child will go to university

Mother hopes other for child

Mother-child interactions: Education (scale)

Mother-child interactions: Non-educational (scale)

\section{Child's attitudes and behaviours}

Ability beliefs (scale)

Locus of control (scale)

Enjoyment of school (intrinsic values, scale)

School results important in life (extrinsic values)

Hobbies/interests important in life

Material possessions important in life

Anti-social behaviours (scale)

Hyperactivity (scale)

Emotional symptoms (scale)

Conduct problems (scale)

Experience of bullying (scale)

Pro-social behaviours (scale)

Peer problems (scale)

Participation in leisure/out-of-school activities

Teacher-child relations (scale)

Observations

Adjusted R-squared

\section{Regression coefficient}

(1)

(2)

$\begin{array}{ll}-0.3 & -0.3 \\ -0.3 & -0.5\end{array}$

$\begin{array}{cc}1.1^{* * *} & 0.5^{* *} \\ 0.2 & 0.2\end{array}$

Omitted

$\begin{array}{cc}8.3^{* * *} & 3.3^{* * *} \\ 13.7^{* * *} & 5.7^{* * *} \\ 6.7^{* *} & 3.0^{* * *} \\ & \\ -4.7^{* * *} & -0.8^{* *} \\ -1.4^{* *} & -1.0^{* *}\end{array}$

$3.4^{* * *}$

$1.3^{* * *}$

$4.0 * * *$

$2.2^{* * *}$

$2.0 * * *$

0.3

$6.1^{* * *}$

$2.0^{* * *}$

$1.9 * * *$

$1.3^{* * *}$

$-3.1 * * *$

$-2.1^{* * *}$

$-3.6^{* * *}$

$-1.5^{* * *}$

$-4.5^{* * *}$

$-1.5^{* * *}$

0

$-0.1$

$-1.3^{* * *}$

$-0.9 * * *$

$-1.4^{* *}$

$-1.1 * * *$

$-1.8 * * *$

$-0.8 * * *$

$0.7^{* *}$

$0.5^{* *}$

$2.3^{* * *}$

0.7

$-0.1$

$-0.4$

7972

7972

0.439

0.703

Notes: table contains selected coefficients from two OLS regression models of the determinants of Key Stage 2 (age 11) percentile score. Missing dummies are included, where appropriate, but their coefficient estimates are not shown. Both models also include controls for SEP quintile, demographic and family background characteristics, and primary school characteristics. The second model (shown on the right) additionally controls for attainment at Key Stage 1 (age 7). All other coefficient estimates can be found in Appendix A3. ${ }^{* *},{ }^{* *}$, and ${ }^{*}$ indicate significance at the 1,5 and $10 \%$ levels respectively.

Whilst Table 2 showed how socially graded each factor is in the population (i.e. the strength of its association with SEP), Table 3 tells us about the strength of the association between individual mediating variables and children's attainment at age 11. Next we can combine the two pieces of information to provide a summary decomposition of the relative importance of each factor, in 
accounting for the attainment gaps as described in the overview paper. Table 4 shows the results of this exercise. We focus our results on the difference in mean test scores, between the poorest $20 \%$ of children and those in the middle $20 \%$ of the SEP distribution (the middle-bottom gap), which is a summary measure of differences between those in poverty and average children. In contrast, the difference between the poorest and the richest $20 \%$ (the top-middle gap) is an indicator of the full extent of educational inequality at 11 . The left panel of Table 4 presents results from the levels model, and the right panel presents results from the value-added model. For each set of results, we show the contribution of a particular factor to the raw middle-bottom and top-bottom gaps, first in terms of percentile point Key Stage 2 scores, and then as a percentage of the total raw gap.

The top panel gives an overall summary of the decompositions. Of the 14.3 percentile point middle-bottom SEP gap in level scores, we can explain 10.9 (76\%) using all our measured distal and proximal factors, leaving 3.4 percentile points (24\%) - unexplained. And of the 31.3 percentile point topbottom gap, 26.8 points (86\%) are explained and 4.5 points (14\%) unexplained. The right panel shows that the conditioning on Key Stage 1 increases the proportion of the middle-bottom gap that is explained to $87 \%$. To a large extent this displaces the predictive power of child and family characteristics $-66 \%$ is attributed to prior ability, leaving $21 \%$ to other measured factors associated with the widening socio-economic gap. We see a similar pattern for the top-bottom gap, with $59 \%$ explained by prior ability differences, $34 \%$ attributed to other measured factors and just $7 \%$ unaccounted for. Hence in the value-added models, the factors considered explain the widening attainment gap, between the poorest and most affluent, somewhat more fully than they do the gap between the poorest and middle income children.

Table 4. Breakdown of the bottom-middle and bottom-top SEP gaps in average Key Stage 2 scores

\begin{tabular}{|c|c|c|c|c|c|c|c|c|}
\hline \multirow{4}{*}{ Factor } & \multicolumn{8}{|c|}{ Difference from Q1 attributed to factor } \\
\hline & \multicolumn{4}{|c|}{ (1) Levels model } & \multicolumn{4}{|c|}{ (2) Value-added model } \\
\hline & \multicolumn{2}{|c|}{ Percentile } & \multicolumn{2}{|c|}{ As \% of total } & \multicolumn{2}{|c|}{ Percentile } & \multicolumn{2}{|c|}{ As $\%$ of total } \\
\hline & Q3 & Q5 & Q3 & Q5 & Q3 & Q5 & Q3 & Q5 \\
\hline All & 14.33 & 31.33 & $100 \%$ & $100 \%$ & 14.33 & 31.33 & $100 \%$ & $100 \%$ \\
\hline All measured factors (sum I to VIII) & 10.9 & 26.82 & $76.1 \%$ & $85.6 \%$ & 12.53 & 29.13 & $87.4 \%$ & $93.0 \%$ \\
\hline Residual unexplained component & 3.43 & 4.51 & $23.9 \%$ & $14.4 \%$ & 1.80 & 2.20 & $12.6 \%$ & $7.0 \%$ \\
\hline I. Key Stage 1 & - & - & - & - & 9.44 & 18.45 & $65.9 \%$ & $58.9 \%$ \\
\hline II. Parental education & 2.98 & 7.83 & $20.8 \%$ & $25.0 \%$ & 1.32 & 3.97 & $9.2 \%$ & $12.7 \%$ \\
\hline Mother's education & 1.40 & 3.86 & 9.8 & 12.3 & 0.60 & 2.20 & 4.2 & 7.0 \\
\hline Father's education & 1.58 & 3.97 & 11.0 & 12.7 & 0.73 & 1.77 & 5.1 & 5.6 \\
\hline III. Demographic ch & 0.80 & 1.31 & $5.6 \%$ & $4.2 \%$ & 0.06 & 0.32 & $0.4 \%$ & $1.0 \%$ \\
\hline Female & 0.00 & 0.00 & 0.0 & 0.0 & 0.03 & 0.08 & 0.2 & 0.2 \\
\hline Non-white & 0.13 & 0.13 & 0.9 & 0.4 & 0.11 & 0.11 & 0.8 & 0.4 \\
\hline Family structure & -0.21 & -0.24 & -1.4 & -0.8 & -0.40 & -0.49 & -2.8 & -1.6 \\
\hline Month of birth & -0.18 & -0.05 & -1.3 & -0.2 & 0.02 & 0.00 & 0.1 & 0.0 \\
\hline Mother's age at birth & 0.30 & 0.38 & 2.1 & 1.2 & 0.16 & 0.36 & 1.1 & 1.1 \\
\hline Number older siblings & 0.22 & 0.45 & 1.5 & 1.4 & 0.06 & 0.17 & 0.5 & 0.5 \\
\hline Number younger siblings by 9 & -0.03 & -0.08 & -0.2 & -0.2 & -0.02 & -0.03 & -0.1 & -0.1 \\
\hline Twin & 0.00 & 0.01 & 0.0 & 0.0 & 0.00 & -0.01 & 0.0 & 0.0 \\
\hline English second language at 11 & 0.00 & 0.00 & 0.0 & 0.0 & 0.00 & 0.00 & 0.0 & 0.0 \\
\hline Parental employment at age 4 & 0.82 & 0.97 & 5.7 & 3.1 & 0.20 & 0.24 & 1.4 & 0.8 \\
\hline Parental health at age 4 & -0.24 & -0.25 & -1.7 & -0.8 & -0.10 & -0.11 & -0.7 & -0.4 \\
\hline
\end{tabular}


( Table 4 cont'd)

IV. School composition and quality

Mean pupil KS1 (std score)

Mean VA KS1-2 (std score)

Proportion pupils FSM

V. Pre-school environments
Birth weight
Gestation
Breast feeding
Smoking in pregnancy
Post-natal depression
HLE
Read to daily at 3
Regular sleeping routine at 3
Centre-based care pre-3
Nursery age 3 to 4

VI. Parent's attitudes and behaviours

Mother found school valuable

Maternal locus of control

Mother hopes at least $1 \mathrm{~A}$-level

Mother hopes university

Mother hopes other for child

Mother-ch interactions: ed

Mother-ch interactions: non-ed

VII. Child attitudes and behaviours
Ability beliefs
Locus of control
Enjoyment of school
School results important in life
Hobbies important in life
Possessions important in life
Anti-social behaviours
Hyperactivity
Emotional symptoms
Conduct problems (scale)
Experience of bullying (scale)
Pro-social behaviours
Peer problems (scale)
Teacher-child relations (scale)
Leisure activities (scale)

VIII. Missing flags

$\begin{array}{cccccccc}\mathbf{1 . 7 6} & \mathbf{4 . 3 9} & \mathbf{1 2 . 3 \%} & \mathbf{1 4 . 0 \%} & \mathbf{- 0 . 0 3} & \mathbf{1 . 4 6} & -\mathbf{0 . 2 \%} & \mathbf{4 . 6 \%} \\ 1.65 & 2.85 & 11.5 & 9.1 & -0.77 & -1.33 & -5.4 & -4.2 \\ 0.80 & 2.50 & 5.6 & 8.0 & 1.00 & 3.14 & 7.0 & 10.0 \\ -0.69 & -0.96 & -4.8 & -3.1 & -0.26 & -0.36 & -1.8 & -1.1 \\ & & & & & & & \\ \mathbf{0 . 4 9} & \mathbf{1 . 1 0} & \mathbf{3 . 4 \%} & \mathbf{3 . 5 \%} & \mathbf{- 0 . 1 9} & -\mathbf{- 0 . 0 3} & \mathbf{- 1 . 3 \%} & -\mathbf{0 . 1 \%} \\ 0.18 & 0.22 & 1.3 & 0.7 & 0.05 & 0.06 & 0.3 & 0.2 \\ 0.00 & -0.01 & 0.0 & 0.0 & 0.00 & -0.01 & 0.0 & 0.0 \\ 0.24 & 0.68 & 1.7 & 2.2 & 0.25 & 0.69 & 1.7 & 2.2 \\ -0.37 & -0.50 & -2.6 & -1.6 & -0.25 & -0.34 & -1.7 & -1.1 \\ -0.07 & -0.08 & -0.5 & -0.3 & -0.08 & -0.09 & -0.5 & -0.3 \\ 0.71 & 1.20 & 5.0 & 3.8 & 0.04 & 0.08 & 0.3 & 0.3 \\ -0.32 & -0.50 & -2.2 & -1.6 & -0.21 & -0.32 & -1.5 & -1.0 \\ 0.11 & 0.16 & 0.8 & 0.5 & 0.00 & 0.00 & 0.0 & 0.0 \\ -0.01 & -0.04 & 0.0 & -0.1 & -0.01 & -0.04 & 0.0 & -0.1 \\ 0.01 & -0.04 & 0.0 & -0.1 & 0.01 & -0.07 & 0.1 & -0.2 \\ & & & & & & & \\ \mathbf{2 . 6 1} & \mathbf{6 . 4 6} & \mathbf{1 8 . 2 \%} & \mathbf{2 0 . 6 \%} & \mathbf{1 . 1 1} & \mathbf{2 . 6 9} & \mathbf{7 . 7 \%} & \mathbf{8 . 6 \%} \\ 0.06 & 0.13 & 0.4 & 0.4 & 0.05 & 0.11 & 0.4 & 0.3 \\ 0.54 & 1.02 & 3.8 & 3.3 & 0.24 & 0.45 & 1.7 & 1.4 \\ 0.14 & -0.64 & 1.0 & -2.0 & 0.06 & -0.26 & 0.4 & -0.8 \\ 1.95 & 6.03 & 13.6 & 19.3 & 0.81 & 2.52 & 5.7 & 8.0 \\ -0.06 & -0.42 & -0.4 & -1.3 & -0.03 & -0.19 & -0.2 & -0.6 \\ 0.03 & 0.33 & 0.2 & 1.1 & 0.01 & 0.06 & 0.0 & 0.2 \\ -0.05 & 0.00 & -0.3 & 0.0 & -0.03 & 0.00 & -0.2 & 0.0 \\ & & & & & & & \\ \mathbf{2 . 7 4} & \mathbf{6 . 1 4} & \mathbf{1 9 . 1 \%} & \mathbf{1 9 . 6 \%} & \mathbf{1 . 0 7} & \mathbf{2 . 5 4} & \mathbf{7 . 4 \%} & \mathbf{8 . 1 \%} \\ 0.13 & 0.64 & 0.9 & 2.0 & 0.05 & 0.25 & 0.3 & 0.8 \\ 0.33 & 1.43 & 2.3 & 4.6 & 0.18 & 0.78 & 1.3 & 2.5 \\ 0.04 & 0.04 & 0.3 & 0.1 & 0.01 & 0.01 & 0.0 & 0.0 \\ 0.80 & 1.25 & 5.6 & 4.0 & 0.27 & 0.42 & 1.9 & 1.3 \\ 0.26 & 0.47 & 1.8 & 1.5 & 0.17 & 0.3 & 1.2 & 1.0 \\ -0.24 & -0.44 & -1.7 & -1.4 & -0.16 & -0.3 & -1.1 & -1.0 \\ 0.17 & 0.28 & 1.2 & 0.9 & 0.08 & 0.12 & 0.5 & 0.4 \\ 0.89 & 1.41 & 6.2 & 4.5 & 0.31 & 0.48 & 2.1 & 1.5 \\ 0.00 & 0.00 & 0.0 & 0.0 & 0.01 & 0.02 & 0.1 & 0.1 \\ 0.31 & 0.39 & 2.2 & 1.2 & 0.23 & 0.28 & 1.6 & 0.9 \\ 0.07 & 0.13 & 0.5 & 0.4 & 0.06 & 0.1 & 0.4 & 0.3 \\ -0.11 & 0.05 & -0.7 & 0.2 & -0.05 & 0.02 & -0.3 & 0.1 \\ -0.17 & -0.2 & -1.2 & -0.6 & -0.12 & -0.14 & -0.8 & -0.5 \\ -0.01 & 0.00 & 0.0 & 0.0 & -0.02 & -0.02 & -0.2 & -0.1 \\ 0.23 & 0.68 & 1.6 & 2.2 & 0.07 & 0.21 & 0.5 & 0.7 \\ & & & & & & & \\ -\mathbf{0 . 4 9} & -\mathbf{0 . 4 1} & -\mathbf{3 . 4 \%} & -\mathbf{1 . 3 \%} & -\mathbf{0 . 2 5} & -\mathbf{0 . 2 6} & \mathbf{- 1 . 7 \%} & -\mathbf{0 . 8 \%}\end{array}$

The main body of the table shows how the numbers in the 'All measured factors' row can be broken down into different components. The first line shows that differences in parental education between deprived and more advantaged children can account for about 3 (8) percentile points of the
Key Stage 2 gap between bottom and middle (top) SEP quintile children, or about $21 \%(25 \%)$ of the total gap. This is the contribution of parental education over and above any influence through the mediating proximal influences we discuss further on. When we consider progress between 7 
and 11 , we see that parental education accounts for increases in the gaps of 1.3 and 4.0 percentile points between the lowest SEP and the two higher SEP groups, or $9 \%$ and $13 \%$ respectively of the total gaps. Parental education differences between socio-economic groups are clearly the most important factor in explaining the gaps in attainment at 7 , and the gaps in progress during the primary school years. So it represents about a third of the educational inequality at age 11 , net of prior attainment at age 7 . The huge importance of parental education is particularly notable because, as noted previously, these numbers relate only to the direct effects of parental education, or the part that is not explained by any of the other (more proximal) variables in the model.

All other demographic influences contribute relatively little to the attainment gaps, although the younger age of mothers of disadvantaged children, and larger family size, have some explanatory power. So, lone parenthood, numbers of siblings, ethnicity, etc contribute little to attainment gaps in terms of total attainment at age 11 or progress since 7. Schools do matter, especially the school value-added measure between ages 7 and 11 for child progress, as might be expected. Our measures of parental attitudes, behaviours and beliefs explain in the region of a fifth of the gradients in the levels model, and around $8 \%$ when prior ability is held constant. Interestingly, children's views and behaviours independently explain a similar proportion to parents', in both the levels and valueadded models, with their importance more than halved when Key Stage 1 is controlled. Unfortunately, without earlier measures of the key variables, we cannot distinguish whether success at Key Stage 1 leads to improvements in children's and parents' outlooks, or whether persistence in these factors means that causation runs the other way from attitudes and behaviours to achievement at 7 . Differences in pre-school environments can account for little of the gaps. Hence parental attitudes and beliefs have an influence that is not far away from that of parental education (not transmitted by proximal factors) in driving the social gradient in attainment at age 11 and progress since age 7 . Taken together, parent and child attitudes, beliefs and behaviours and school quality, explain over half of the attainment gaps between the most and least affluent children at 11 in the levels model. They explain around one-fifth in the value-added model, or about half of the 13 percentile point gap that emerges between 7 and 11 .

The method used here allows us to be more specific about the key drivers. Differences in maternal aspirations for university alone account for $19 \%$ of the top-bottom gap in levels terms and $8 \%$ in the value-added model. Assuming causality, equalising aspirations for university across socioeconomic groups would narrow the Key Stage 2 gap by 6.0 percentile points or, if we assume that performance at 7 is fixed and determined by other factors, by 2.5 percentile points. Mother's locus of control and attitudes to education, play a smaller but not trivial supporting role in making parental attitudes and beliefs such important predictors of child outcomes.

The four dimensions of child behavioural problems - anti-social, hyperactivity, emotional symptoms and conduct problems - together account for $9.6 \%$ (6.6\%) of the middle- (top-) bottom gap in level scores and $4.3 \%(2.9 \%)$ of the middle- (top-) bottom gap in value-added scores, a substantial amount relative to the other child-level variables. Of these, the greater emotional symptoms of disadvantaged children play little explanatory role, whereas hyperactivity and conduct problems are the most important. Similarly, it is the beliefs that school results and hobbies and interests are important in life, that drive the beneficial effects of the educational values of higher-income children, and a more internal locus of control, rather than a strong belief in their own ability, that drives the explanatory power of child self-concept.

Altogether, just the four factors of: maternal aspirations for university; hyperactivity; child locus of control; and the belief that school results are important in life, can account for $32 \%$ and $28 \%$ of the overall top-bottom and middle-bottom SEP gaps respectively. When prior ability is held constant, the equivalent proportions are $13 \%$ and $11 \%$ of the overall gaps, or around a third of the 5 and 13 percentile point gaps that open up between 7 and 11 among children from different socio-economic groups who started the period with the same Key Stage 1 performance.

\section{Conclusions}

It has long been known that children from more deprived backgrounds, achieve less well in terms of education attainment, than their more affluent 
peers. More recently it has been shown that these attainment gaps start early in life and continue to widen through childhood (see Feinstein 2003 and 2004). Furthermore the extent of these gradients is not constant through time and has recently been diminishing (Gregg and Macmillan 2010). This research, with its two companion studies (Dearden et al 2010; Chowdry et al 2010) aims to show the extent of these gradients and give a sense of when in childhood they emerge for the most recent cohort data available. The main thrust of this study, is to explore the contribution that parent and child attitudes, beliefs and aspirations, make toward these gaps, and their emergence in the primary school years.

A number of features stand out from the study as worthy of note. First, around one third of the large educational gaps that are apparent at age 11 emerge through the primary school years. A sizable portion of these attainment gaps are associated with other aspects of families that differ by social background, especially parental education and also school quality. Parental education, which is obviously related to variations in economic circumstances within families, offers an important and distinct source of education attainment gradients. Hence as we consider the contribution of parental and child aspirations, beliefs and behaviours in driving the socio-economic gradients educational attainment, it is also important to note their role in driving gradients by parental education as well by SEP.

Parental aspirations and attitudes to education vary particularly strongly with socio-economic position. $81 \%$ of the richest mothers say they hope their 9-year-old will go to university, compared with only $37 \%$ of the poorest mothers. There are also large differences according to whether the mother found school valuable for themselves. Children's attitudes and behaviours in primary school vary in the degree to which they are socially graded. Poor children tend to view themselves as scholastically less able, are less likely to believe school results are important in life, and exhibit higher levels of hyperactivity, conduct problems and peer problems. However, their levels of school enjoyment and cooperative behaviour differ little from those of more affluent children. The attitudes, beliefs, and behaviours of parents and children have a major role to play in accounting for the gaps that remain unexplained. Together they explain around half of the social gradient in educational attainment at age 11 when prior ability is not controlled, and around one-sixth when prior ability is held constant. Given that about $60 \%$ of the gaps in the latter model are explained solely by differences in outcomes at age 7 , the figure of onesixth of the total translates into around half of the increase in the gradient over the period. Hence these factors act as major transmission mechanisms for how both social disadvantage itself, and parental education, impact on educational attainment. The adverse attitudes to education of disadvantaged mothers are one of the single most important factors associated with the children's deficits at age 11. In particular, 9-year-olds whose mothers hope they will go to university, will score 2.5 percentile points higher at age 11 than the child of the parent with the lowest aspirations, given the same prior attainment and parental education etc. This factor alone explains a fifth of the test score gap between the richest and poorest children at 11 , for given attainment at age 7. Likewise greater behavioural problems of disadvantaged children are the second key factor in accounting for their poorer educational outcomes. We find evidence that children with high levels of anti-social behaviours, hyperactivity and conduct problems at the ages of 8 to 9, scored lower at Key Stage 1, but even taking this into account, such behaviours appear to interfere with the learning process between 7 and 11. Other types of behaviour problems do not appear to play the same role. Yet whilst parental aspirations and behaviour problems are of key importance, a whole range of adverse attitudes, behaviours and beliefs contribute to the educational deficits of low income children. Other factors we identify as important are the lack of a sense of personal efficacy (both of mothers and their children), and the view that school results are not important in life.

The fact that parental aspirations and attitudes to education for their children and the child's own attitudes and behaviours are important to educational attainment in the primary school years, has a number of important policy implications. First, attempts to raise school results and progression through to university for reasonably able children from poorer families needs to start before secondary school starts. Furthermore, parents expectations and attitudes need to be shifted, not just those of the children. Poorer parental attitudes 
do appear to reflect in part their own experiences of education, which were often negative, but they may also be substantially out of date in the educational opportunities open to middle ability children these days, with a third of children attending university. The pre-school environment is influential on attainment at 7 years, but perhaps unsurprisingly, it plays little role in learning development through primary school. This suggests that our measures are not just reflecting an underlying marker of good parenting that drives attainment throughout childhood, as early markers of parenting predict early outcomes but not later progress.

\section{Acknowledgements}

The authors gratefully acknowledge funding from the Joseph Rowntree Foundation (JRF), the Leverhulme Trust and the ESRC Large Grant RES-060-23-0011. We would also like to thank Lindsey Macmillan for research assistance to all the members of our advisory groups. All errors remain the responsibility of the authors.

\section{References}

Chowdry H, Crawford C and Goodman A. (2010) The role of attitudes and behaviours in explaining socioeconomic differences in attainment at age 16. Longitudinal and Life Course Studies, 2, (59-76).

Currie J and Stabile M. (2006) Child mental health and human capital accumulation: The case of ADHD. Journal of Health Economics, 25, 1094-1118.

Dearden L, Sibieta L and Sylva K. (2010) The socio-economic gradient in early child outcomes: evidence from the Millennium Cohort Study. Longitudinal and Life Course Studies, 2, 19-40.

Feinstein L. (2003) Inequality in the Early Cognitive Development of British Children in the 1970 Cohort. Economica, 70, 73-98.

Feinstein L. (2004) Mobility in Pupils' Cognitive Attainment During School Life. Oxford Review of Economic Policy, 20, No. 2: Education.

Goodman R. (1997) The Strengths and Difficulties Questionnaire: A Research Note. Journal of Child Psychology and Psychiatry, 38, 581-586.

Goodman A, Gregg P and Washbrook L. (2010) Children's educational attainment and the aspirations, attitudes and behaviours of parents and children through childhood. Longitudinal and Life Course Studies, 2, 1-18.

Gregg P and Macmillan L. (2010) Family income, education and cognitive ability in the next generation: exploring income gradients in education and test scores for current cohorts of youth. Longitudinal and Life Course Studies, 1, 259-280.

Harter S. (1985) Manual for the self-perception profile for children. University of Denver: Denver, CO.

McLeod J and Kaiser K. (2004) Childhood Emotional and Behavioral Problems in Educational Attainment. American Sociological Review, 69, 636-658.

NICHD Early Child Care Research Network and Duncan G. (2003). Modeling the Impacts of Child Care Quality on Children's Pre-school Cognitive Development Child Development, 74, 1454-1475.

Wigfield A and Eccles JS. (2000) Expectancy-Value Theory of Achievement Motivation. Contemporary Educational Psychology, 25, 68-81.

\section{Endnotes}

\footnotetext{
'Avon was a former county area containing around 0.5 million residents in Bristol and the surrounding area in the West of England. It is now divided into four separate Local Authorities.

ii For information on ALSPAC, see http://www.bris.ac.uk/alspac.

iii The children in the ALSPAC cohort were age 3 to 4 between 1994 and 1996, before the introduction of guaranteed free half-day places.
} 